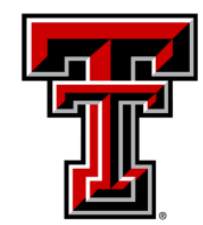

TEXAS TECH UNIVERSITY

Libraries"

\title{
EFFECTS OF ONCOMIng VehicLe Size ON OVERTAKING JUdGMENTS
}

\section{The Texas Tech community has made this publication openly available. Please share how this access benefits you. Your story matters to us.}

\begin{tabular}{|l|l|}
\hline Citation & $\begin{array}{l}\text { Samuel J. Levulis, Patricia R. DeLucia, Jason Jupe, Effects of } \\
\text { oncoming vehicle size on overtaking judgments, In Accident } \\
\text { Analysis \& Prevention, Volume 82, 2015, Pages 163-170, ISSN 0001- } \\
4575, \text { https://doi.org/10.1016/i.aap.2015.05.024. }\end{array}$ \\
\hline Citable Link & $\underline{\text { http://hdl.handle.net/2346/73443 }}$ \\
\hline Terms of Use & $\underline{\text { CC BY-NC-ND 4.0 }}$ \\
\hline
\end{tabular}


8

9

17

18

19

20

21

22

23

\section{Effects of Oncoming Vehicle Size on Overtaking Judgments} Samuel J. Levulis ${ }^{1}$, Patricia R. DeLucia ${ }^{*}$, Jason Jupe ${ }^{2}$

${ }^{1}$ Texas Tech University, MS 2051, Lubbock, TX 79409-2051, U.S.A., samuel.levulis@ttu.edu, pat.delucia@ttu.edu

${ }^{2}$ Armstrong Forensics, 8500 Shoal Creek Blvd \#201b, Austin, TX 78757-7528, U.S.A. Present Address: Rimkus Consulting Group, Inc., 3410 Far West Blvd, Suite 375 Austin, Texas 7873115 3194, jjupe@rimkus.com

*Corresponding author, 806-834-0968 


\section{Abstract}

During overtaking maneuvers on two-way highways drivers must temporarily cross into the opposite lane of traffic, and may face oncoming vehicles. To judge when it is safe to overtake, drivers must estimate the time-to-contact (TTC) of the oncoming vehicle. Information about an oncoming vehicle's TTC is available in the optical expansion pattern, but it is below threshold during high-speed overtaking maneuvers, which require a large passing distance. Consequently, we hypothesized that drivers would rely on perceived distance and velocity, and that their overtaking judgments would be influenced by oncoming vehicle size. A driving simulator was used to examine whether overtaking judgments are influenced by the size of an oncoming vehicle, and by whether a driver actively conducts the overtaking maneuver or passively judges whether it is safe to overtake. Oncoming motorcycles resulted in more accepted gaps and false alarms than larger cars or trucks. Results were due to vehicle size independently of vehicle type, and reflected shifts in response bias rather than sensitivity. Drivers may misjudge the distances of motorcycles due to their relatively small sizes, contributing to accidents due to right-of-way violations. Results have implications for traffic safety and the potential role of driver-assistance technologies.

Keywords: Overtaking, Gap acceptance, Right-of-way violation, Motorcycles, Size-arrival effect, Time-to-collision

2 


\section{Introduction}

\subsection{Overtaking}

In 2012, there were 91,000 accidents in the United States in which one vehicle passed

50 another vehicle (including overtaking), resulting in 672 deaths and 19,000 injuries (NHTSA,

51 2012). To avoid a collision with an oncoming vehicle, the driver who is overtaking presumably

52 must estimate how much time is left before a collision would occur with that vehicle.

53 Specifically, the driver must judge whether the time required to overtake the lead vehicle (TRO)

54 is shorter than the time it will take the oncoming vehicle to arrive at the location where the driver

55 will re-enter the right lane, referred to as time-to-contact (TTC; Gray \& Regan, 2005). This is

56 represented in Figure 1, based on analyses by Gray and Regan (2005), who identified the

57 physical variables that are necessary to determine whether a temporal gap between an overtaking

58 driver and an oncoming vehicle is large enough to complete an overtaking maneuver. The

59 purpose of the current study is to examine whether overtaking judgments are influenced by the

60 size of an oncoming vehicle, and by whether a driver actively conducts the overtaking maneuver

61 or passively judges whether it is safe to overtake. 


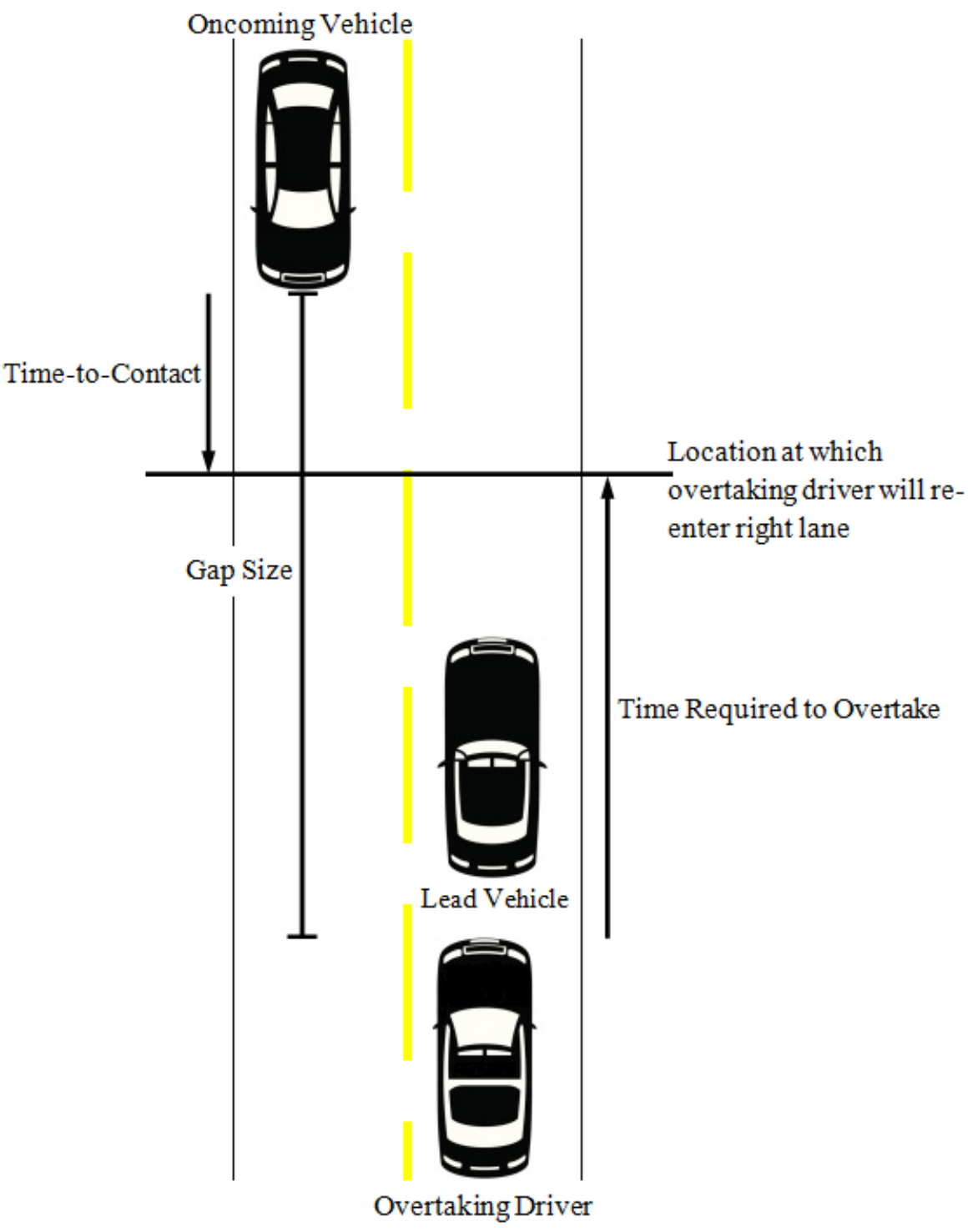

63 Figure 1. Representation of the physical variables that must be considered when determining

64 whether a gap is large enough for an overtaking maneuver. Adapted from "Perceptual Processes

65 Used by Drivers During Overtaking in a Driving Simulator” by R. Gray and D. M. Regan, 2005, Human Factors, 47, p. 409. Copyright 2005 by the Human Factors and Ergonomics Society. 
maneuver is not possible and a collision will occur if an overtaking maneuver is attempted, unless one of the vehicles takes evasive action.

An equation provided by Gordon and Mast (1970) can be used to determine the difference between TTC and TRO. This equation is based on an analysis of actual overtaking performance, and predicts the distance required to overtake (DRO, in meters) at various speeds:

$$
D R O=34.20+2.88 \mathrm{~V}+.01094 \mathrm{~V}^{2}
$$

where V represents the driver's (and lead vehicle's) initial velocity in $\mathrm{km} / \mathrm{h}$. This method of estimating DRO has been used in previous research (Gray \& Regan, 2005) and is employed here.

As the overtaking vehicle's velocity increases, DRO also increases. The TRO can be calculated by dividing the DRO by the overtaking vehicle's average velocity across the maneuver, estimated by the overtaking vehicle's initial speed and its acceleration profile. The TTC of the oncoming vehicle can be derived by dividing DTC (distance to contact-the oncoming vehicle's distance from the location where the overtaking driver re-enters the right lane) by the oncoming vehicle's average velocity across that distance. If TTC is greater than TRO, overtaking is possible.

\subsection{Visual information available for overtaking judgments}

It is evident from the preceding analysis that it is important for drivers to accurately estimate TTC during an overtaking maneuver. It has been proposed that individuals can estimate TTC (the time remaining before an approaching object reaches they eye) on the basis of an optical invariant, tau (e.g., Lee, 1976). Tau is the instantaneous ratio of an approaching object's visual angle and the rate of change of that angle. Tau provides accurate information about TTC (assuming certain conditions are met, e.g., the object is rigid and moves at a constant velocity) and does not require perceptual estimates of distance or velocity. 
However, even when tau is available, TTC judgments can be influenced by less reliable information, Of particular relevance to this study is the effect of size on such judgments: A small near approaching object can appear to arrive later than a large far approaching object that is specified by tau to arrive later, known as the size-arrival effect (DeLucia, 1991; DeLucia \& Warren, 1994; Smith et al., 2001). For example, when participants used a driving simulator to estimate when an approaching vehicle would reach their intersection during a left-turn scenario, mean TTC judgments generally decreased as the approaching vehicle size increased (e.g., motorcycle vs delivery van; Caird \& Hancock, 1994). Similarly, when participants viewed videos of vehicles that approached a traffic intersection, filmed from the view of a driver waiting on the non-priority road, estimates of when the approaching vehicles would arrive at a tarmac strip in front of the driver's location were greater for motorcycles than larger vehicles (Horswill et al., 2005).

These results suggest that vehicle size can influence TTC judgments when tau is available. Importantly, it is unlikely that tau is available during most high-speed overtaking scenarios because of the large distances involved (Gould et al., 2013). Tau is effective only when an approaching object's rate of optical expansion is above the threshold for the detection of expansion, which is between about .003 and .006 radians per second (Muttart et al., 2005). In contrast, an oncoming vehicle's rate of optical expansion is below this threshold during a typical overtaking maneuver.

When tau is not effective, it is reasonable to expect drivers to base overtaking judgments on other information. We considered the possibility that drivers rely on the oncoming vehicle's apparent distance and velocity (Cavallo \& Laurent, 1988; Smeets et al., 1996). In this case, judgments would be expected to be influenced by depth cues such as relative size (e.g., 
115 Hochberg, 1986), and consequently the size-arrival effect (e.g., Horswill et al., 2005). Smaller

116 vehicles would appear farther, and to arrive later, than larger vehicles.

117

118

119

120

121

122

123

124

125

126

127

128

129

130

131

132

133

134

135

136

137

\subsection{Motorcycle safety}

Effects of size on TTC judgments have implications for motorcycle safety, which are over-represented in accident statistics (NHTSA, 2012). In 2012, 4,957 motorcyclists were killed and another 93,000 were injured in the United States, representing a 7\% and 15\% increase, respectively, compared to 2011 (NHTSA, 2014). Moreover, in 2012, motorcycles made up only $3 \%$ of the registered vehicles and accounted for only $0.7 \%$ of all vehicle miles travelled, but represented $15 \%$ of the fatalities and $4 \%$ of the injuries (NHTSA, 2014). Hurt et al. (1981) reported that compared to large and heavyweight motorcycles, smaller motorcycles are overrepresented in traffic accidents, but noted that this finding could be due to factors other than size per se. In the current study, we tease apart the effect of size from vehicle type on overtaking judgments.

The most frequent accident scenario involving a motorcycle and another moving vehicle is one in which the vehicle violates the motorcycle's right-of-way (Hurt et al., 1981; Pai, 2011). In this type of accident, the vehicle travels into the motorcycle's path, for example, when turning left in front of the motorcycle or overtaking in front of it. In a review of right-of-way accidents involving motorcycles, Pai (2011) identified two major causes of such accidents: detection errors, in which a motorist fails to see a motorcycle, and decision errors, in which the motorist detects the motorcycle but nevertheless performs an unsafe driving maneuver.

Detection errors suggest that motorcycles have low conspicuity. For example, accident risk can be reduced when a motorcyclist increases conspicuity by using daytime headlights and wearing reflective or fluorescent clothing (Hurt et al., 1981; Wells et al., 2004), though the 
effectiveness of such conspicuity treatments may depend on the scene that constitutes the background of the motorcycle (Pai, 2011). Other factors that can affect a driver's ability to detect an oncoming motorcycle include expectancy and the motorist's experience with motorcycles (Hurt et al., 1981; Pai, 2011). However, ensuring detection is not sufficient to eliminate accidents. Even when motorists detect a motorcycle, they must decide whether there is sufficient time to complete a driving maneuver without colliding with the motorcycle. Errors in decisionmaking account for a substantial number of motorcycle accidents (Pai, 2011). The current study focuses on overtaking—specifically, on gap-acceptance decisions that occur after an oncoming vehicle has been detected. We hypothesized that the size of an oncoming vehicle would influence overtaking judgments, and that drivers would accept a greater number of gaps for small oncoming vehicles than large oncoming vehicles. Improving the accuracy of overtaking judgments would increase safety. To improve accuracy in overtaking judgments, it is important to identify whether errors are due to a motorist's inability to discriminate between safe and unsafe gaps or to a greater likelihood of choosing one response over the other. These response characteristics can be measured with sensitivity and response bias in the framework of signal detection theory (Stanislaw \& Todorov, 1999), which we use here.

\subsection{Effects of feedback on overtaking judgments}

Judging whether a gap is acceptable during overtaking is difficult for drivers. Observations of drivers on an English highway indicated that 14\% of overtaking maneuvers were initiated when gap size was insufficient, resulting mostly in lane sharing (overtaking while partially in both lanes) and cutting in (re-entering the original lane with an unsafe headway in front of the overtaken vehicle) (Wilson \& Best, 1982). Furthermore, when participants judged 
161 the last safe moment at which an overtaking maneuver could be initiated in the face of an

162 oncoming vehicle, more than $30 \%$ of responses were too late-when there was insufficient time

163 to safely pass (Jones \& Heimstra, 1964). In a similar task, in which drivers estimated the last

164 moment at which they could pass a lead car and still return back to the right lane before reaching

165 a specified location in the road, the differences between the drivers' estimates and their actual

166 performance ranged from 20\% to 50\% (Gordon \& Mast, 1970).

167

168

169

170

171

172

173

174

175

176

177

178

179

180

181

182

183

Judgment errors in the studies by Jones and Heimstra (1964), and Gordon and Mast (1970), may have occurred, in part, because participants did not receive feedback on their overtaking decisions. This is supported by a study of overtaking in which on-road participants first estimated the last safe moment to pass a lead car in front of an oncoming vehicle (Lucas et al., 1973) and then made the same judgments using filmed scenes. Participants who were given feedback on judgment accuracy showed significant improvement in subsequent on-road judgments, as compared to a group who did not receive the feedback. The importance of feedback was shown more recently in a driving simulator study in which participants made more unsafe passive overtaking judgments than they did active overtaking maneuvers (Gray \& Regan, 2005). During passive trials, participants reported overtaking decisions by pressing a button whereas during active trials participants completed the overtaking maneuver and thus received visual feedback from their actions. The implication for transportation safety is that drivers' overtaking decisions may improve after completing active training (i.e., getting visual feedback) in a driving simulator. Consequently, we included active and passive conditions in our study and expected that active participants would make safer overtaking judgments than passive participants.

\section{Experiment 1}


185

186

187

188

189

190

191

192

193

194

195

196

197

198

199

200

201

202

203

204

205

206

influenced by the size of an oncoming vehicle, and by whether a driver actively conducts the overtaking maneuver or passively judges whether it was safe to overtake. Because optical expansion is below threshold during high-speed overtaking, putatively leading drivers to use depth cues to judge distance, we anticipated that drivers would be more likely to accept a gap as safe for overtaking when the oncoming vehicle was a motorcycle rather than a larger car or truck. The gap acceptance paradigm is commonly employed (Kirkby \& Stroud, 1978; Pai, 2011) and has been characterized as the most direct test of a driver's likelihood of accepting a gap in different traffic scenarios (Sager et al., 2014). We also expected safer judgments during active overtaking maneuvers than passive judgments because active control provides visual feedback.

\subsection{Method}

\subsubsection{Participants}

Twenty-four college students (12 male, 12 female) between 17 and 35 years of age $(M=$ 21.83, $S D=3.91$ ) participated for course credit in an introductory psychology course. Participants were recruited through an online sign-up system. All held a driver's license for between 1 and 9 years $(M=4.32, S D=2.11)$ and reported normal or corrected visual acuity, and normal hearing and motor control. None currently or previously held a motorcycle license.

\subsubsection{Apparatus}

Traffic scenes were created with a STISIM Drive ${ }^{\mathrm{TM}}$ simulator housed in a $2.40 \mathrm{GHz}$ computer. STISIM Drive ${ }^{\mathrm{TM}}$ is a medium-fidelity driving software package (Tilak et al., 2008; Tippey et al., 2014), and is widely used to evaluate driving performance (Freund et al., 2002; 2005; Lee et al., 2003; Shechtman, 2010). Data collected with STISIM Drive ${ }^{\mathrm{TM}}$ have been shown to correlate with data collected during on-road testing (Bédard et al., 2010; Lee et al., 2003; 
Shechtman, 2010). A Logitech force-feedback steering wheel and floor-mounted throttle and brake pedals were used by participants to drive the simulated vehicle. Sound was also used to provide road noise, engine acceleration and deceleration, and tire screeches. STISIM Drive ${ }^{\mathrm{TM}}$ has been characterized as a driving simulator that responds to driver inputs and presents realistic traffic scenes in real time (Freund et al., 2005).

Scenes were presented in 1280 x 1024 pixel resolution on a flat panel LCD monitor subtending $68^{\circ}$ diagonally, and were updated at 30 frames/s. Levers on the steering wheel allowed participants to rotate their viewing positions, which simulated looking over one's shoulder while changing lanes.

\subsubsection{Displays}

Scenes depicted a car-following scenario on a straight, two-lane, two-way highway (see Figure 2). Each lane was $4.27 \mathrm{~m}$ wide and the lanes were divided by a dashed yellow line. The speed limit was posted with a $65-\mathrm{mph}$ sign $(104.61 \mathrm{~km} / \mathrm{h})$. At the start of each scene, the participant's vehicle traveled $16.76 \mathrm{~m}$ behind a lead car (white Dodge Intreped) in the right lane. The steering and speed of the participant's vehicle were automatically controlled; speed matched that of the lead car. An oncoming vehicle was visible, and consisted of a motorcycle, a town car, or a large delivery truck. The vehicle widths were $0.76 \mathrm{~m}, 2.13 \mathrm{~m}$, and $2.59 \mathrm{~m}$ for the motorcycle, car, and truck, respectively. Seven seconds after the start of the scene, a 1000-Hz auditory tone signaled participants to make their decision. The oncoming vehicle was visible for the entire approach. In contrast to Gray and Regan (2005), the oncoming vehicle's rate of optical expansion, and thus tau, was always below previous threshold estimates (Muttart et al., 2005) when the tone occurred. 


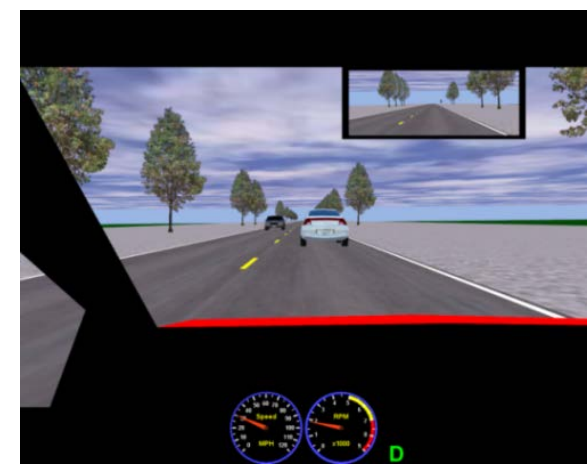

Figure 2. A screen shot of a scene.

\subsubsection{Procedure}

Participants viewed displays from $41.13 \mathrm{~cm}$. First, they were familiarized with the handling, acceleration, and braking capabilities of the simulated vehicle by passing lead cars with maximum acceleration on a two-lane highway without oncoming vehicles. Then participants practiced the overtaking task with five trials that presented a safe overtaking opportunity. Participants were randomly assigned to the active or passive condition. In both conditions, participants were told that their car would initially follow a lead car on a two-lane, two-way highway, and that an oncoming vehicle would be present in the opposite lane.

\subsubsection{Active condition}

Participants were informed that, at the beginning of each scene, the steering and speed of their vehicle would be automatically controlled, and that the speedometer on their simulated dashboard would display their current speed. They were told that they would get complete control of their vehicle when a tone occurred and that they should pass the lead car if they felt it was safe to do so. They were further instructed to drive the simulated car as they would on a real highway, obey the rules of the road, and avoid accidents.

\subsubsection{Passive condition}


The passive condition was identical to the active condition except that participants were

248 informed that the steering and speed of their vehicle would be controlled for the duration of each

249 scene. They were told that, when the tone occurred, they should report whether they felt it was

250 safe to pass the lead car, using buttons on the steering wheel.

Feedback on decision accuracy was not provided, but active participants received visual

252 feedback when they maneuvered the vehicle. Participants in both conditions completed four 253 blocks of 27 trials, each separated by a rest period. The entire experiment lasted approximately 25470 minutes.

255

256

257

258

259

260

261

262

263

264

265

266

267

268

269

\subsubsection{Design}

Our main variable of interest was oncoming vehicle size. To include both safe gaps and unsafe gaps, we created different temporal gap sizes at the time the tone occurred. We did this by manipulating the speed of the participant's car (and lead car; $48.28 \mathrm{~km} / \mathrm{h}, 64.37 \mathrm{~km} / \mathrm{h}, 80.47$ $\mathrm{km} / \mathrm{h})$ and the speed of the oncoming vehicle $(72.42 \mathrm{~km} / \mathrm{h}, 88.51 \mathrm{~km} / \mathrm{h}, 104.61 \mathrm{~km} / \mathrm{h})$. In addition, the distance between the oncoming vehicle and the front of the participant's vehicle was either $457.20 \mathrm{~m}$ or $609.60 \mathrm{~m}$ at the time of the tone. These manipulations resulted in nine safe gaps (TTC - TRO >0) and nine unsafe gaps (TTC - TRO <0). Both safe and unsafe gaps occurred within each level of the manipulated parameters so that accurate overtaking judgments could not be achieved on the basis of any one of these variables (e.g., distance). Each temporal gap was presented twice for each vehicle size, resulting in 108 trials. The order of trials was counterbalanced across participants. The largest optical expansion rate of the oncoming vehicle when the tone signaled the participant to respond was $0.0009 \mathrm{rad} / \mathrm{s}$. This is well below previous estimates of expansion detection threshold.

\subsubsection{Analyses}


Data from 24 of the 2592 total trials (0.93\%) were removed due to a software bug that

271

272

273

274

275

276

277

278

279

280

281

282

283

284

285

286

287

288

289

290

291

292

prevented the tone from being presented. We separately analyzed the percentage of accepted gaps and false alarms, and signal detection theory measures of sensitivity ( $\left.d^{\prime}\right)$ and response bias $(\beta)$. For passive participants, an accepted gap occurred when they reported it was safe to pass. For active participants, an accepted gap occurred when their vehicle crossed the center line. A false alarm occurred when a participant accepted a gap when a safe overtaking maneuver was not possible (TTC - TRO <0).

To further understand the effect of oncoming vehicle size on overtaking decisions, we analyzed $d^{\prime}$ ' and $\beta$. In each trial, a signal (safe gap) was either present (TTC - TRO $>0$ ) or absent (TTC - TRO $<0$ ), and the participant indicated that the signal was either present (accepted the gap) or absent (rejected the gap). When the hit rate or false alarm rate for a given subject and vehicle was 0.00 or 1.00 it was replaced with 0.01 and 0.99 , respectively (DeLucia \& Novak, 1997).

The hit rate and false alarm rate were used to calculate $d$ ' and $\beta$ for each subject and vehicle size. A value of 0 for $d$ ' indicates an inability to discriminate between safe and unsafe gaps. Relatively larger $d^{\prime}$ values indicate relatively greater sensitivity. A value of 1 for $\beta$ indicates no bias: the participant reported that the gap was safe equally as often as reporting that the gap was unsafe. $\beta$ values above 1 indicate a conservative criterion: participants' judgments were biased toward rejecting the gap—reporting that it was unsafe (Stanislaw \& Todorov, 1999). $\beta$ values below 1 indicate a liberal criterion: participants' judgments were biased toward accepting the gap-reporting that it was safe.

Analyses focused on our primary variables of interest - the size of the oncoming vehicle (motorcycle, car, truck), and control type (active, passive). Each dependent variable was 
293

294

295

296

297

298

299

300

301

302

303

304

305

306

307

308

309

310 311 type was not significant.

312 tests with alpha set to .05.

\subsection{Results}

\subsubsection{Accepted gaps}

\section{Table 1}

\subsubsection{False alarms}

analyzed with a 2 (control type: active, passive) x 3 (vehicle size: motorcycle, car, truck) mixed analysis of variance (ANOVA) with control type as a between-groups factor. The same pattern of results occurred when we analyzed the arcsine transformation of the percentage of accepted gaps and false alarms. Significant main effects of vehicle size were followed up with Tukey HSD

Results are summarized in Table 1, and indicated a main effect of vehicle size on the percentage of accepted gaps. Tukey HSD tests indicated that participants accepted a larger percentage of gaps in front of motorcycles than cars and trucks. The difference between cars and trucks also was significant. The main effect of control type was not significant.

Main effects of oncoming vehicle size in Experiment 1

\begin{tabular}{|c|c|c|c|c|c|c|c|}
\hline \multirow[b]{2}{*}{ Effect } & \multicolumn{3}{|c|}{ Oncoming vehicle size $M(S D)$} & \multirow[b]{2}{*}{$F$} & \multirow[b]{2}{*}{$d f$} & \multirow[b]{2}{*}{$p$} & \multirow[b]{2}{*}{$\eta_{p}^{2}$} \\
\hline & Motorcycle & Car & Truck & & & & \\
\hline Accepted gaps (\%) & 63.15 (29.89) & 43.55 (25.97) & 31.90 (23.59) & 52.06 & 2,44 & .0001 & 0.70 \\
\hline False alarms (\%) & $24.93(16.04)$ & 12.76 (10.95) & $7.87(9.26)$ & 33.73 & 2,44 & .0001 & 0.61 \\
\hline$d^{\prime}$ & $1.13(0.59)$ & $1.27(0.75)$ & $1.12(0.74)$ & 0.56 & 2,44 & .5740 & 0.03 \\
\hline$\beta$ & $0.92(1.08)$ & 2.45 (3.85) & $2.70(3.36)$ & 3.22 & 2,44 & .0496 & 0.13 \\
\hline
\end{tabular}

The main effect of vehicle size on the percentage of false alarms was also significant. Participants exhibited a larger percentage of false alarms in front of motorcycles than cars and trucks. The difference between cars and trucks was not significant. The main effect of control

\subsubsection{Signal detection theory measures}


Results indicated that mean $d$ ' was not affected by either vehicle size or control type.

314 The main effect of vehicle size on $\beta$ was significant. However, Tukey HSD tests did not indicate 315 any significant pairwise differences among vehicle sizes. The main effect of control type was not 316 significant.

317

\subsection{Discussion}

Our hypothesis that participants would be more likely to accept a gap as safe for overtaking when the oncoming vehicle was a motorcycle rather than a car or large truck was supported. The percentages of accepted gaps and false alarms were higher for motorcycles than for either cars or trucks. Our signal detection theory analyses suggest that these differences were not due to changes in sensitivity for the different vehicles, consistent with the idea that tau was below threshold. Results of response bias were ambiguous; the overall F-test showed a main effect of vehicle size, but the perhaps too conservative Tukey tests did not indicate any significant pairwise differences among vehicles.

Our hypothesis that overtaking judgments would be safer for participants who actively conduct overtaking maneuvers than for those who passively judge whether it is safe to overtake was not supported. Effects of control type were not significant for any of our dependent measures. This result is not consistent with those of Gray and Regan (2005), who reported that overall performance was worse for passive overtaking judgments: participants made unsafe overtaking maneuvers on a smaller percentage of active trials than they did unsafe overtaking judgments in passive trials. This discrepancy may be partly due to differences in visual information provided in the two studies. Whereas Gray and Regan (2005) reported presenting some scenes in which tau was above threshold, in the current study, tau was always below 
threshold at the moment the overtaking decision occurred. The ineffective expansion information in our study may have reduced the benefits of feedback from active control.

\section{Experiment 2}

Results of Experiment 1 suggest that the size of an oncoming vehicle influences overtaking judgments. However, we cannot rule out the effect of vehicle type, which covaried with size as it does in the real world. It may be that drivers were using a simple rule to pass in front of motorcycles but not delivery trucks (e.g., perhaps trucks are seen as more harmful than motorcycles). To rule out this interpretation, we conducted Experiment 2 to tease apart the effects of vehicle size and type. Using 3D modeling software, we reduced the size of the original delivery truck until it was the same size as the motorcycle. Similarly, we increased the size of the original motorcycle until it was the same size as the original delivery truck. This allowed for comparisons among a small motorcycle, small delivery truck, large motorcycle, and large delivery truck. If vehicle size is the basis for the results of Experiment 1, we should find differences between the small and large vehicles regardless of whether they are motorcycles or trucks. If vehicle type underlies the results, we should find differences between the motorcycle and truck regardless of their sizes.

\subsection{Method}

\subsubsection{Participants}

Twenty-four college students (12 male, 12 female) between 18 and 48 years of age ( $M=$ 21.88, $S D=7.24$ ) participated for course credit in an introductory psychology course. Participants were recruited through an online sign-up system.. All held a driver's license for between 0.3 and 30 years $(M=5.30, S D=5.66)$ and reported normal or corrected visual acuity, and normal hearing and motor control. None currently or previously held a motorcycle license. 


\subsubsection{Displays and procedure}

The apparatus, displays, procedure, and design were as described in Experiment 1 except for modifications necessary to accommodate a fourth oncoming vehicle. We used Google SketchUp, a 3D modeling program, to alter the sizes of the original motorcycle and delivery truck to produce two sets of vehicles that were identical in size. Specifically, the delivery truck from Experiment 1 was reduced in size until it matched that of the motorcycle. The motorcycle's size was increased until it matched that of the delivery truck. Because the original vehicles did not have identical width-to-height ratios we also slightly altered their dimensions to produce vehicles of equal size that did not appear distorted on the width or height dimensions. The final vehicle dimensions were as follows: small motorcycle and small truck: $1.10 \mathrm{~m}$ (width) by $1.52 \mathrm{~m}$ (height); large motorcycle and large truck: $3.20 \mathrm{~m}$ (width) by $4.42 \mathrm{~m}$ (height). As in Experiment 1, each of the 18 temporal gaps was presented twice for each vehicle. This resulted in a total of 144 experimental trials. The entire experiment lasted just under 90 minutes.

\subsubsection{Analyses}

Analyses were identical to Experiment 1, except that we had four oncoming vehicles. Each dependent variable was analyzed with a 2 (control type: active, passive) x 4 (oncoming vehicle: small motorcycle, small truck, large motorcycle, large truck) mixed ANOVA with control type as a between-groups factor. Due to violations of sphericity, probability values for all effects of the oncoming vehicle reflect Greenhouse-Geisser corrections.

\subsection{Results}

\subsubsection{Accepted gaps}

Results are summarized in Table 2, and indicated a main effect of the oncoming vehicle on the percentage of accepted gaps. Participants accepted a larger percentage of gaps in front of 
small motorcycles and small trucks than either large motorcycles or large trucks. The differences between small motorcycles and small trucks and between large motorcycles and large trucks were not significant. The main effect of control type was not significant.

\section{Table 2}

Main effects of the oncoming vehicle in Experiment 2

\begin{tabular}{|c|c|c|c|c|c|c|c|c|}
\hline \multirow[b]{2}{*}{ Effect } & \multicolumn{4}{|c|}{ Oncoming vehicle $M(S D)$} & \multirow[b]{2}{*}{$F$} & \multirow[b]{2}{*}{$d f$} & \multirow[b]{2}{*}{$p$} & \multirow[b]{2}{*}{$\eta_{\mathrm{p}}^{2}$} \\
\hline & $\begin{array}{c}\text { Small } \\
\text { motorcycle }\end{array}$ & $\begin{array}{l}\text { Small } \\
\text { truck }\end{array}$ & $\begin{array}{c}\text { Large } \\
\text { motorcycle }\end{array}$ & $\begin{array}{l}\text { Large } \\
\text { truck }\end{array}$ & & & & \\
\hline Accepted gaps (\%) & $79.16(16.64)$ & 72.09 (23.48) & 20.53 (23.49) & 23.49 (25.74) & 119.17 & 3,66 & .0001 & 0.84 \\
\hline False alarms (\%) & 33.61 (12.33) & $29.74(16.22)$ & $5.22(9.10)$ & $7.22(11.09)$ & 87.87 & 3,66 & . 0001 & 0.80 \\
\hline$d^{\prime}$ & $0.92(0.69)$ & $0.89(0.87)$ & $0.91(0.74)$ & $0.82(0.69)$ & 0.09 & 3,66 & .9138 & 0.00 \\
\hline$\beta$ & $0.58(0.36)$ & $1.59(2.75)$ & $4.14(4.58)$ & $3.58(4.34)$ & 6.12 & 3,66 . & .0026 & 0.22 \\
\hline
\end{tabular}

Note. $p$ values reflect Greenhouse-Geisser corrections

\subsubsection{False alarms}

The main effect of the oncoming vehicle on the percentage of false alarms was significant. Participants exhibited a larger percentage of false alarms in front of small motorcycles and small trucks than large motorcycles and large trucks. Just as for the percentage of accepted gaps, the differences between small motorcycles and small trucks, and between large motorcycles and large trucks, were not significant. The main effect of control type was not significant.

\subsubsection{Signal detection theory measures}

The main effect of the oncoming vehicle on mean d' was not significant. The main effect of control type also was not significant. The main effect of oncoming vehicle on mean $\beta$ was significant. Mean $\beta$ was greater for large motorcycles than for either small motorcycles or small trucks, and was greater for large trucks than small motorcycles. Differences between large trucks 
400 and large motorcycles, and between small motorcycles and small trucks, were not significant.

401 The main effect of control type was not significant.

402

403

404

405

406

407

408

409

410

411

412

413

414

415

416

417

418

419

420

421

422

\subsection{Discussion}

In Experiment 2, participants accepted more gaps and exhibited more false alarms in front of small motorcycles and small delivery trucks than they did in front of larger versions of the same vehicles. The implication is that participants did not base their judgments on vehicle type (and associations such as differential harm), but rather used vehicle size as a primary cue. These findings support our interpretation of the results from Experiment 1. Furthermore, our analyses of $d^{\prime}$ and $\beta$ suggest that the differences in overtaking behavior among the different vehicles was due to changes in response bias rather than sensitivity.

\section{General discussion}

The purpose of the current study was to determine whether overtaking judgments are influenced by the size of an oncoming vehicle, and by whether a driver actively conducts an overtaking maneuver or passively judges whether it is safe to overtake. We hypothesized that the size of an oncoming vehicle would influence overtaking decisions because rate of optical expansion is below threshold during high-speed overtaking, putatively leading participants to rely on perceived distance and velocity. Results of Experiment 1 supported this hypothesis: participants accepted more gaps as safe, and committed more false alarms, when the oncoming vehicle was a motorcycle than a car or large truck, consistent with previous reports of the sizearrival effect on driving-related judgments (Caird \& Hancock, 1994; Horswill et al., 2005). demonstrated that results of vehicle size —apart from vehicle type, affected overtaking decisions. Results of Experiment 2 suggested further that effects of size on overtaking decisions were due 
423 to changes in response bias rather than sensitivity. Participants were more biased towards 424 rejecting a gap as unsafe when the oncoming vehicle was a large motorcycle or large truck 425 compared to smaller versions of these vehicles. conduct overtaking maneuvers (and thus receive visual feedback) than for those who passively 428 judge whether it is safe to overtake was not supported. Effects of control type were not 429 significant for any of our dependent measures in either experiment. This result is not consistent 430 with those of Gray and Regan (2005), who reported that performance was worse for passive 431 participants.

432

433

434 435 436 437 438 439 440 441 442 443 of-way.

\subsection{Vehicle size}

In Experiment 1, participants exhibited almost twice as many accepted gaps and more than three times as many false alarms when the oncoming vehicle was a motorcycle compared to a delivery truck. Drivers may be particularly susceptible to effects of size during high-speed overtaking because optical expansion (and thus tau) is below threshold, presumably leading them to rely on less reliable heuristic information. Whether observers rely on accurate information such as tau, or heuristics such as relative size, during TTC judgments may depend on the distance of an approaching object and thus the quality of optical motion information such as expansion (DeLucia, 2008). Accepting more gaps in front of motorcycles than larger vehicles would increase a driver's risk of collision with motorcycles, and this effect of vehicle size may contribute to the relatively high rate of accidents in which a vehicle violates a motorcycle's right-

Our results regarding vehicle size both corroborate and extend studies of size in earlier studies. For example, effects of oncoming vehicle size on TTC judgments were demonstrated in 
446 both left-hand turn (Caird \& Hancock, 1994) and merging (Horswill et al., 2005) scenarios.

447 Consistent with these studies, we obtained an effect of size on overtaking. However, these 448 studies measured TTC estimations with a prediction-motion task. In this task, participants press a 449 button to indicate the TTC of an approaching object after it has disappeared from view. This

450

451

452

453

454

455

456

457

458

459

460

461

462

463

464

465

466

467

method has been criticized as being artificial (Hancock \& Manser, 1997). In the current study, the oncoming vehicle was always visible and (active) participants performed an overtaking task. In addition, our study employed scenes with self-motion, whereas Horswill et al. (2005), and Caird and Hancock (1994) only used object (vehicle) motion. Compared to object motion alone, the addition of forward self-motion can increase the threshold for object motion detection (Probst et al., 1987) and can result in shorter and less accurate TTC estimates (Gray \& Regan, 2000). The current study showed that the effects of size obtained with a prediction-motion task and object motion in prior studies generalize to a more realistic context that included self-motion, an oncoming vehicle that was always visible, and an active task.

\subsection{Control type}

Unexpectedly, the difference between active and passive conditions was not significant in either Experiment 1 or 2, which is not consistent with Gray and Regan (2005). A potentially important difference between studies was that Gray and Regan reported presenting some scenes in which optical expansion, and thus tau, was above threshold. In our study, optical expansion was always below threshold at the moment of the overtaking decision, which may have reduced the benefits of feedback from active control. Consequently, reliable information may not have been available to support perceptual learning. It would be worthwhile to determine whether drivers could benefit from other sources of information when tau is not available. This may 
468 include lateral or downward motion of the vehicle in the driver's visual field (e.g., Sager et al., 469 2014).

A second possible contributor to the discrepant findings may be display differences. In Gray and Regan’s (2005) passive trials, all vehicles except the participant’s vehicle disappeared when the overtaking response was made. Consequently, participants did not see the entire approach of the oncoming vehicle in the passive trials, as they did in the active trials. In the latter, participants received additional visual information from the oncoming vehicle, potentially resulting in better performance. In contrast, in our study, active and passive participants saw the entire approach of the oncoming vehicle, reducing the differences between groups. Future research should investigate the conditions under which different forms of feedback influence performance. In addition to viewing the oncoming vehicle's approach, other sources of feedback might include information about an oncoming vehicle's absolute TTC or whether overtaking was actually safe on a given trial.

Finally, another possible source of the discrepancy between the results of Gray and Regan (2005) and the current study pertains to the transition from automated to manual control. In Gray and Regan’s study, participants completed a series of 10 overtaking maneuvers during each active trial, and maintained control of their vehicle for the duration of the trial. In our study, when active participants decided to overtake, they only completed a single overtaking maneuver during a trial, and were given control of the vehicle seven seconds after the scene's onset. This transition from automated to manual control may have made the active task more difficult than the passive task and should be investigated in future studies.

\subsection{Limitations}


Although our results indicate that oncoming vehicle size influences overtaking

491

492

493

494

495

496

497

498

499

500

501

502

503

504

505

506

507

508

509

510

511

512 judgments, the current study has limitations that warrant further research. For example, the overtaking scenarios used in the current study were less complex than those typically encountered in real-world driving: the current study employed straight sections of roadway in good weather and visibility, and vehicles that behaved predictably and travelled at constant speeds. However, in the real world, characteristics of roadway geometry, weather, visibility, and driver behavior are much more variable. In addition, the use of a simulator may have produced biases in decision-making, in which participants took more risks than they would have in the real world (Gray \& Regan, 2005). Even if such biases occur, it is reasonable to expect that they would occur in the same manner under all conditions of oncoming vehicle size. That is, we aimed to measure the relative rather than the absolute effects of size on overtaking judgments. Indeed, studies comparing an on-road assessment to results generated from STISIM Drive ${ }^{\mathrm{TM}}$ exhibited such relative validity (Shechtman et al., 2009; Wang et al., 2010). In other words, our finding that motorcycles resulted in a greater percentage of accepted gaps than cars and trucksthe rank ordering of conditions - can be expected to occur in the real traffic environment, although the absolute percentage in each condition may not be the same as in the real world.

A further limitation of the current study is in the method we employed to determine gap safety. Specifically, gaps were defined as safe or unsafe a priori, using a formula derived from previous overtaking performance (Gordon \& Mast, 1970), rather than with the participants' actual data. This was necessary because we did not have performance data for passive participants and needed an equivalent method for determining gap safety across the two conditions. Consequently, if a participant in our active condition accepted a gap as safe but failed to successfully execute the maneuver (e.g., because they lost control of the vehicle), their 
513 judgment would have been correct. In other words, the current study focused on driver

514 perception and the decision to overtake, rather than on vehicle control during overtaking.

515

516

517

518

519

520

521

522

523

524

525

526

527

528

529

530

531

532

533

534

535

\subsection{Practical implications}

Inaccurate judgments of temporal gap size during overtaking can have negative consequences for transportation safety. Accepting unsafe gaps increases the risk of collision with an oncoming vehicle. Rejecting safe gaps can result in traffic delays and decreased highway throughput. Reducing both types of errors has practical value. One possible remedy is to provide drivers with technological aids, for example, an “overtaking assistant” that provides drivers with information about when it is safe to overtake. Prototypes of driver-assistance systems to support overtaking maneuvers have been developed (e.g., Chiang et al., 2014; Hageman et al., 2007), but are not yet commercially available. Other interventions that improved a motorist's ability to detect a motorcycle or judge its approach include increasing a motorcycle’s apparent size by employing alternative fairings or front lighting configurations (Gould et al., 2012; Mortimer \& Schuldt, 1980; Williams \& Hoffman, 1979), and instructing motorcycle riders to travel in the right portion of their lane, which may provide the overtaking driver with more motion cues (Sager et al., 2014). To the extent that the current results generalize to real-world traffic, it is worthwhile to pursue such measures to reduce the incidence of collisions in which a vehicle violates a motorcycle’s right-of-way.

Furthermore, effects of size on response bias during overtaking judgments suggest that such judgments are malleable. The implication is that training may ameliorate the size-arrival effect and improve overtaking judgments. Training via visual feedback alone may not be effective, implicated by the absence of control-type effects in the current study. Providing drivers with experience riding motorcycles (e.g., via driving simulations) is worthy to consider: drivers 
536 with such experience appear to be less frequently involved in crashes in which a vehicle violates

537 a motorcycle's right-of-way (Hurt et al., 1981). Similarly, drivers involved in motorcycle-car

538 crashes who hold a motorcycle license are less likely to be at fault than those without a 539 motorcycle license (Magazzu et al., 2006). This may be due to differences in perceptual 540 strategies: drivers with motorcycle licenses attended more to motorcycles (Shahar et al., 2012) 541 and were better at hazard perception (Horswill \& Helman, 2003), compared to drivers without 542 motorcycle licenses. judgments. 


\section{Acknowledgments}

We thank Trevor Hill, Neil Imbery, Marian Nelson, and Jeffrey Payne for assistance with data collection, and Jeffrey Payne for help with stimulus development.

\section{References}

Bédard, M., Parkkari, M., Weaver, B., Riendeau, J., \& Dahlquist, M. (2010). Assessment of driving performance using a simulator protocol: Validity and reproducibility. American Journal of Occupational Therapy, 64, 336-340.

Caird, J. K., \& Hancock, P. A. (1994). The perception of arrival time for different oncoming vehicles at an intersection. Ecological Psychology, 6, 83-109.

Cavallo, V., \& Laurent, M. (1988). Visual information and skill level in time-to-collision estimation. Perception, 17, 623-632.

Chiang, H.-H., Chen, Y.-L., Wu, B.-F., \& Lee, T. T. (2014). Embedded driver-assistance system using multiple sensors for safe overtaking maneuver. IEEE Systems Journal, 8, 681-698.

DeLucia, P. R. (1991). Pictorial and motion-based information for depth perception. Journal of Experimental Psychology: Human Perception and Performance, 17, 738-748.

DeLucia, P. R. (2005). Does binocular disparity or familiar size information override effects of relative size on judgements of time to contact?. The Quarterly Journal of Experimental Psychology, 58, 865-886.

DeLucia, P. R. (2008). Critical roles for distance, task, and motion in space perception: Initial conceptual framework and practical implications. Human Factors, 50, 811-820.

DeLucia, P. R. (2013). Effects of size on collision perception and implications for perceptual theory and transportation safety. Current Directions in Psychological Science, 22, 199204. 
DeLucia, P. R., \& Novak, J. B. (1997). Judgments of relative time-to-contact of more than two approaching objects: Toward a method. Perception \& Psychophysics, 59, 913-928.

DeLucia, P. R., \& Warren, R. (1994). Pictorial and motion-based depth information during active control of self-motion: Size-arrival effects on collision avoidance. Journal of Experimental Psychology: Human Perception and Performance, 20, 783-798.

Freund, B., Gravenstein, S., Ferris, R., Burke, B. L., \& Shaheen, E. (2005). Drawing clocks and driving cars: Use of brief tests of cognition to screen driving competency in older adults. Journal of General Internal Medicine, 20, 240-244.

Freund, B., Gravenstein, S., Ferris, R., \& Shaheen, E. (2002). Evaluating driving performance of cognitively impaired and healthy older adults: A pilot study comparing on-road testing and driving simulation. Journal of the American Geriatrics Society, 50, 1309-1310.

Gordon, D. A., \& Mast, T. M. (1970). Drivers' judgments in overtaking and passing. Human Factors,12, 341-346.

Gould, M., Poulter, D. R., Helman, S., \& Wann, J. P. (2012). Judgments of approach speed for motorcycles across different lighting levels and the effect of an improved tri-headlight configuration. Accident Analysis \& Prevention, 48, 341-345.

Gould, M., Poulter, D. R., Helman, S., \& Wann, J. P. (2013). Detection of vehicle approach in the presence of additional motion and simulated observer motion at road junctions. Journal of Experimental Psychology: Applied, 19, 171-184.

Gray, R., \& Regan, D. M. (2005). Perceptual processes used by drivers during overtaking in a driving simulator. Human Factors, 47, 394-417.

Gray, R., \& Regan, D. (2000). Simulated self-motion alters perceived time to collision. Current Biology, 10, 587-590. 
Hancock, P. A., \& Manser, M. P. (1997). Time-to-contact: More than tau alone. Ecological Psychology, 9, 265-297.

Hegeman, G., van der Horst, R., Brookhuis, K. A., \& Hoogendoorn, S. P. (2007). Functioning and acceptance of overtaking assistant design tested in driving simulator experiment. Transportation Research Record: Journal of the Transportation Research Board, 2018, 45-52.

Hochberg, J. (1986). Representation of motion and space in video and cinematic displays. In K. R. Boff, L. Kaufman, \& J. P. Thomas (Eds.), Handbook of perception and human performance (pp. 22-1 to 22-64). New York: Wiley.

Horswill, M. S., \& Helman, S. (2003). A behavioral comparison between motorcyclists and a matched group of non-motorcycling car drivers: Factors influencing accident risk. Accident Analysis \& Prevention, 35, 589-597.

Horswill, M. S., Helman, S., Ardiles, P., \& Wann, J. P. (2005). Motorcycle accident risk could be inflated by a time to arrival illusion. Optometry and Vision Science, 82, 740-746.

Hosking, S. G., \& Crassini, B. (2011). The influence of optic expansion rates when judging the relative time to contact of familiar objects. Journal of Vision, 11, 1-13.

Hurt, H. H., Ouellet, J. V., Thom, D. R., 1981. Motorcycle accident cause factors and identification of countermeasures. Vol. 1: Technical Report (DOT HS-5-01160). Los Angeles: University of Southern California, Traffic Safety Center.

Jones, H. V., \& Heimstra, N. W. (1964). Ability of drivers to make critical passing judgments. Journal of Engineering Psychology, 3, 117-122.

Kirkby, C., \& Stroud, P. G. (1978). The effect of motorcyclists’ high-visibility aids on driver gap acceptance. Traffic Engineering \& Control, 19 401-403. 
Lee, D. N. (1976). A theory of visual control of braking based on information about time-tocollision. Perception, 5, 437-459.

Lee, H. C., Cameron, D., \& Lee, A. H. (2003). Assessing the driving performance of older adult drivers: On-road versus simulated driving. Accident Analysis \& Prevention, 35, 797-803.

López-Moliner, J., Field, D. T., \& Wann, J. P. (2007). Interceptive timing: Prior knowledge matters. Journal of Vision, 7, 1-8.

Lucas, R., Heimstra, N., \& Spiegel, D. (1973). Part-task simulation training of drivers’ passing judgments. Human Factors, 15, 269-274.

Magazzù, D., Comelli, M., \& Marinoni, A. (2006). Are car drivers holding a motorcycle licence less responsible for motorcycle-Car crash occurrence? A non-parametric approach. Accident Analysis \& Prevention, 38, 365-370.

Mortimer, R. G., \& Schuldt, R. C. (1980). Field test evaluation of gap-acceptance of drivers as a function of motorcycle front lighting. Proceedings of the International Motorcycle Safety Conference, vol. II , 945-954.

Muttart, J. W., Messerschmidt, W. F., \& Gillen, L. G. (2005). Relationship between relative velocity detection and driver response times in vehicle following situations. $S A E$ Technical Paper, 01-0427.

National Highway Traffic Safety Administration (NHTSA) (2012). Traffic safety facts 2012: A compilation of motor vehicle crash data from the fatality analysis reporting system and the general estimates system (DOT HS 812032). Washington, DC.

National Highway Traffic Safety Administration (NHTSA) (2014). Traffic safety facts 2012 data: Motorcycles (DOT HS 812035). Washington, DC. 
651 Pai, C.-W. (2011). Motorcycle right-of-way accidents—a literature review. Accident Analysis

652

653

654

655

656

657

658

659

660

661

662

663

664

665

666

667

668

669

670

671

672

673 and Prevention, 43, 971-982.

Probst, T., Krafczyk, S., \& Brandt, T. (1987). Object-motion detection affected by concurrent self-motion perception: Applied aspects for vehicle guidance. Ophthalmic and Physiological Optics, 7, 309-314.

Sager, B., Yanko, M. R., Spalek, T. M., Froc, D. J., Bernstein, D. M., \& Dastur, F. N. (2014). Motorcyclist's lane position as a factor in right-of-way violation collisions: A driving simulator study. Accident Analysis \& Prevention, 72, 325-329.

Shahar, A., van Loon, E., Clarke, D., \& Crundall, D. (2012). Attending overtaking cars and motorcycles through the mirrors before changing lanes. Accident Analysis \& Prevention, 44, 104-110.

Shechtman, O. (2010). Validation of driving simulators. Advances in Transportation Studies (Special Issue), 53-62.

Shechtman, O., Classen, S., Awadzi, K., \& Mann, W. (2009). Comparison of driving errors between on-the-road and simulated driving assessment: A validation study. Traffic Injury Prevention, 10, 379-385.

Smeets, J. B. J., Brenner, E., Trébuchet, S., \& Mestre, D. R. (1996). Is judging time-to-contact based on 'tau’? Perception, 25, 583-590.

Smith, M. R. H., Flach, J. M., Dittman, S. M., \& Stanard, T. (2001). Monocular optical constraints on collision control. Journal of Experimental Psychology: Human Perception and Performance, 27, 395-410.

Stanislaw, H. \& Todorov, N. (1999). Calculation of signal detection theory measures. Behavior Research Methods, Instruments, \& Computers, 31, 137-149. 
674 Tilak, R., Xholi, I., Schowalter, D., Ferris, T., Hameed, S., \& Sarter, N. (2008). Crossmodal links

675

676

677

678

679

680

681

682

683

684

685

686

687

688

689

690

691 in attention in the driving environment: The roles of cueing modality, signal timing, and workload. Proceedings of the Human Factors and Ergonomics Society Annual Meeting, 52, $1815-1819$.

Tippey, K. G., Sivaraj, E., Ardoin, W. J., Roady, T., \& Ferris, T. K. (2014). Texting while driving using Google Glass: Investigating the combined effect of heads-up display and hands-free input on driving safety and performance. Proceedings of the Human Factors and Ergonomics Society Annual Meeting, 58, 2023-2027.

Wang, Y., Mehler, B., Reimer, B., Lammers, V., D'Ambrosio, L. A., \& Coughlin, J. F. (2010). The validity of driving simulation for assessing differences between in-vehicle informational interfaces: A comparison with field testing. Ergonomics, 53, 404-420.

Wells, S., Mullin, B., Norton, R., Langley, J., Connor, J., Lay-Yee, R., \& Jackson, R. (2004). Motorcycle rider conspicuity and crash related injury: Case-control study. British Medical Journal, 328, 857.

Williams, M. J., \& Hoffmann, E. R. (1979). Conspicuity of motorcycles. Human Factors, 21, 619-626.

Wilson, T., \& Best, W. (1982). Driving strategies in overtaking. Accident Analysis \& Prevention, 14, 179-185. 